\title{
107
}

\section{Rainforest Goes School}

\author{
Chair: Helmut Stemmer (AT) \\ Panel: Michaela Picheler (AT), Ingrid Schwarz (AT)
}

Keywords: methodologies, curriculum development, teacher education, ethical and social issues, classroom practice

An important point was to see the rainforest project in relation to other papers compare findings, weaknesses, strengths, doubts, reflections, and so assess our project anew. This highlights the strength of this project, located in complexity and diversity of content, methods and media and reconfirmed that project is on the 'right path', despite of difficulties faced in the past. Concerning theories of learning and teaching, we have seen parallels to other presenters, that point to approaches that have been used successfully in the rainforest project and partly correspond to the methodology of the project taken from international development education called 'Global Learning'.

The paper from Bernard Cornu Winds of change in the teaching profession reflects the very complex role of teachers from today and tomorrow. These ideas describe the role that many teachers adopt in the rainforest project. Further, the demands that are put on the school from society (such as globalisation, democratisation and complexification), also mentioned by Cornu, meet the goals and means of the rainforest project.

The presentation from Lone Dirckinck-Holmfeld about Conceptual design of a virtual learning environment based on problem oriented project pedagogy (POPP) corresponds with the rainforest project in various ways:

- Learning to learn and to see problems and subject matters in a new way

- Critical information handling and teamwork

- Mutual responsibility for own and others learning.

The notion from the professional group about social and ethical issues that 'social and ethical issues are often purely presented to pupils in 
uninteresting ways', is a statement the Austrian rainforest project can respond to and offer a different position and experience. The project provides many possibilities and methodological approaches to learning about, and engaging pupils in, projects around social and ethical issues, which are not boring and uninteresting. It combines traditional methods plus ICT environment so the is enjoyable and interesting to students and teachers.

As often discussed at this conference, there is the difficulty that ICT without greater content is not very appealing to teachers and students. The rainforest project suggests and demonstrates that themes like globalisation, complexity, democratisation, equity and global citizenship can be discussed in schools successfully and combined well with the use of ICT. Students and teachers are interested and motivated to learn about the content and use ICT as medium to transport their messages to a bigger audience. ICT offers a range of methods to engage in these themes nationally and internationally.

Schools have either worked within specific subjects or have included the entire school in the rainforest project. It depends on the school culture, whether they put priority on project-oriented learning or not. In many countries these open learning spaces are barely available even when considered necessary. This ICT project can enhance open learning spaces, yet it also builds on pre-existing school-structures and will not offer miracles. Feedback appreciated that we verbalised that ICT does not save time, but enables better public visibility and offers more continuity.

Various participants have shown great interest in participating in this project and there might be a possibility of expanding it to countries such as Australia, New Zealand, Denmark, Germany, Slowenia and Mexico and thus allowing an international discussion between students about these crucial issues of rainforests and climate change. Some students in countries without rainforest, like Austria, are concerned that they should give advice on the situation of rainforests. "As this is not our intention and never has been, we really want to encourage, in this stage of the project process, students to discuss these issues world-wide, especially with kids and teachers living in or near a rainforest or generally in other countries, instead of proposing simple solutions. These complex problems and issues need a broad basis for discussion, reflection and action."

Another factor that has emerged was that the project bridges soft sciences and hard sciences, which many presenters and participants have asked for and considered as very important. It is an example of a content driven project that reaches out to use ICT as a tool to be more effective and more visible in the world. 